\title{
Rectus Femoris
}

National Cancer Institute

\section{Source}

National Cancer Institute. Rectus Femoris. NCI Thesaurus. Code C53175.

One of the four quadriceps muscles, located in the middle of the front of the thigh. Its actions involve the extension of the knee joint and flexion of the hip. 